Proceedings of the International Symposium on Physics of Materials (ISPMA 14), September 10-15, 2017, Prague

\title{
Twinning Evolution in Magnesium Alloys under Biaxial Loading
}

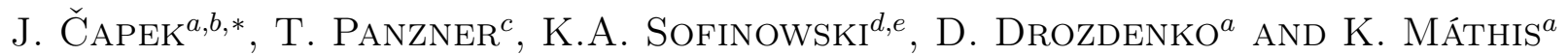 \\ ${ }^{a}$ Charles University, Department of Physics of Materials, Ke Karlovu 5, 12116, Prague 2, Czech Republic \\ ${ }^{b}$ The Czech Academy of Sciences, Nuclear Physics Institute, Rež 130, 250 68, Řež, Czech Republic \\ ${ }^{c}$ Laboratory for Neutron Scattering, NUM, Paul Scherrer Institute, CH-5232 Villigen PSI, Switzerland \\ ${ }^{d}$ Swiss Light Source, Paul Scherrer Institute, CH-5232 Villigen PSI, Switzerland \\ ${ }^{e}$ Ecole Polytech Fed Lausanne, Neutrons \& Xrays Mech Mat, IMX, CH-1012 Lausanne, Switzerland
}

DOI: 10.12693/APhysPolA.134.853

PACS/topics: 61.72.Mm , 43.40.Le, 07.05.Pj, 83.50.-v

\section{Introduction}

Deformation mechanisms of magnesium alloys are an often discussed topic in the scientific community. In addition to basal slip, which is the easiest activated deformation mechanism, deformation twinning and non-basal slip play an important role in plastic deformation. Materials' characteristics, such as the initial texture [1] and grain size [2] as well as the loading path, strongly influence the contribution of these mechanisms to plasticity. The insitu neutron diffraction (ND) method has been successfully utilized for characterization of deformation mechanisms in hexagonal materials. It has been shown that the integrated intensity of particular reflections (parent and twin grain families) is sensitive to the reorientation of the crystal lattice (e.g. due to twinning) during loading. Further, the activity of different slip systems can be deduced from the evolution of the lattice strains with the applied stress and the dislocation density can be evaluated from the change of the peak width. Recently, acoustic emission (AE), another in-situ method, has been used concurrently with neutron diffraction measurements $[3,4]$. $\mathrm{AE}$ technique yields information on deformation twinning nucleation and dislocation glide. The advantage of both methods is to obtain information from entire volume in real time. Further, they provide complementary information: the $\mathrm{AE}$ is sensitive only to twin nucleation, whereas neutron diffraction characterizes twin volume, regardless of nucleation or growth.

The combination of AE and ND measurement was used by several authors and brought completely new information about the deformation behavior of magnesium alloys during uniaxial loading. However, multi-axial testing is also important, since this method better represents the in-service conditions.

*corresponding author; e-mail: jan.capek89@gmail.com

\section{Experimental methods}

$\mathrm{Mg}+1$ wt.\% $\mathrm{Zr}(\mathrm{Mg})$ alloy, having random texture with a grain size of $(100 \pm 10) \mu \mathrm{m}$ was used for the experiments.

The biaxial tests were carried out at POLDI instrument using cruciform specimens. The arms of the samples were $55 \mathrm{~mm}$. The specimen was thinned in the middle in order to ensure uniform deformation in this area. The deformation tests were carried out at room temperature using a load frame at a force control with a rate of $20 \mathrm{~N} / \mathrm{sec}$. In order to collect the ND data, the tests were stopped for approx. $45 \mathrm{~min}$. at predefined force levels. The schematic illustration of the experimental setup is shown at Fig. 1.

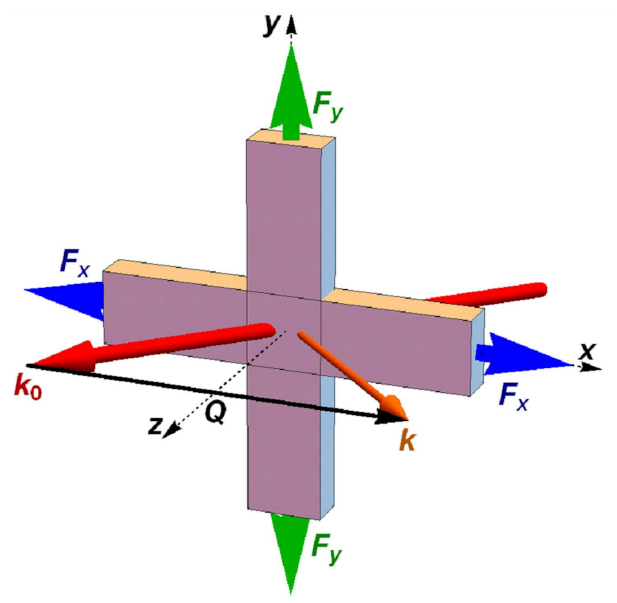

Fig. 1. Schematic illustration of the experimental setup.

The AE testing was performed using a Physical Acoustics PCI- 2 acquisition board and a broadband AE sensor from the same company was mounted on the outside the gauge length using vacuum grease and a pin. The AE was amplified by $40 \mathrm{~dB}$ in the frequency range $100-1200 \mathrm{kHz}$. 
There are several challenges connected to biaxial testing. The evaluation of the stress state at the center of a sample is not straightforward, because the sample does not have well-defined cross-section [5]. Moreover, the circular thickness reduction at the center of specimen causes a so-called "ring-effect" — by compressing the sample along axis $x$ we induce the tensile stress along the axis $y[6]$. Due to this fact the "uniaxial" deformation does not have the radial symmetry along the loading axis. Digital image correlation (DIC) was used to measure the strain field of the sample. Electron backscatter diffraction (EBSD) was used in order to study the microstructure after deformation.

\section{Results}

Samples were deformed equibiaxially $\left(F_{x}=F_{y}\right)$ in tension and compression up to $4 \mathrm{kN}$. The Mises Strain-Force curve is shown in Fig. 2.

Mises strain maps of the center area of the samples after unloading are shown in Fig. 3. It is obvious that although the deformation is not locally homogeneous, it is of the same nature throughout the investigated area. The local inhomogeneity is probably caused by the strong plastic anisotropy of the magnesium.

A detailed view of the deformation can be obtained by separation of the strain into individual components (Fig. 4). Figure 4a shows the results for the equibiaxial tension. The dominant deformation component in this case is the compression along the $z$-axis. At the same time a tensile strain has arisen along the $x$ and $y$ axes. Thus, the deformation has a similar character to a simple uniaxial compression along $z$-axis. Equibiaxial compression has the same character as a simple uniaxial tension along $z$-axis. The dominant deformation component in this case is the elongation along the $z$-axis. At the same time the compressive strain has arisen along the $x$ and $y$ axes.

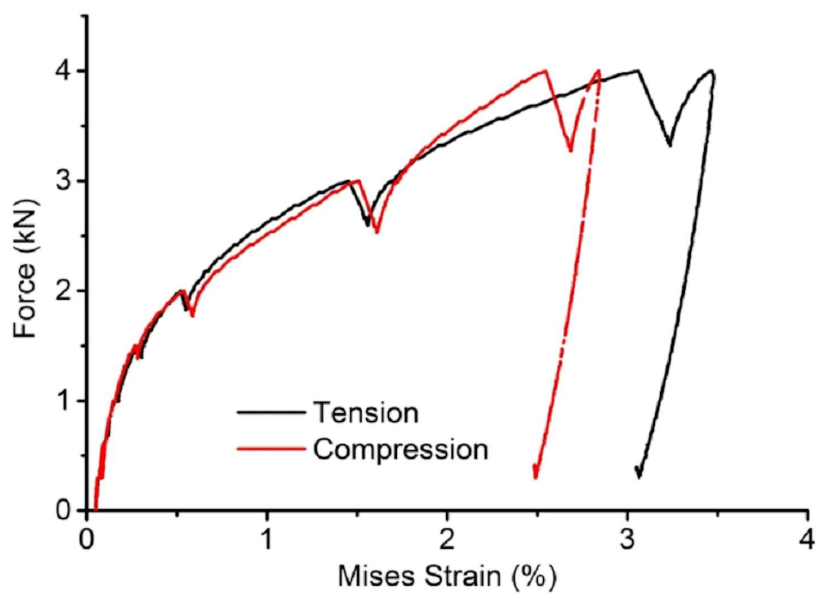

Fig. 2. Strain force deformation curve.
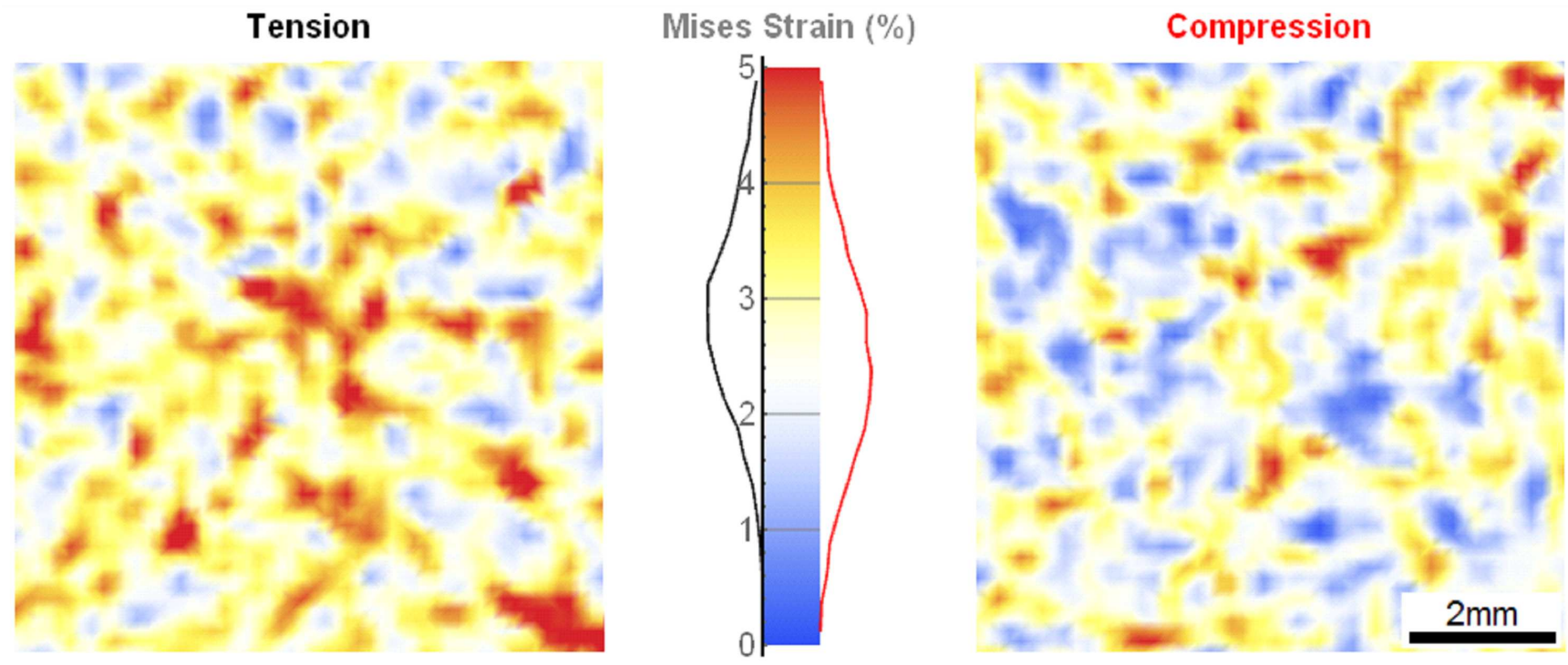

Fig. 3. DIC strain maps of Mises strain after unloading.

In order to reveal the active deformation mechanisms, the AE response was analyzed. Cumulative counts during straining are shown in Fig. 5a. It is slightly larger for the compression than for the tension. The cumulative energy of AE signal is significantly larger for compression (Fig. 5b). Since the twin nucleation is the main source of $\mathrm{AE}$ in magnesium, the results suggest that twin nucleation is more pronounced for compression than for tension and the nucleated twins are larger. 

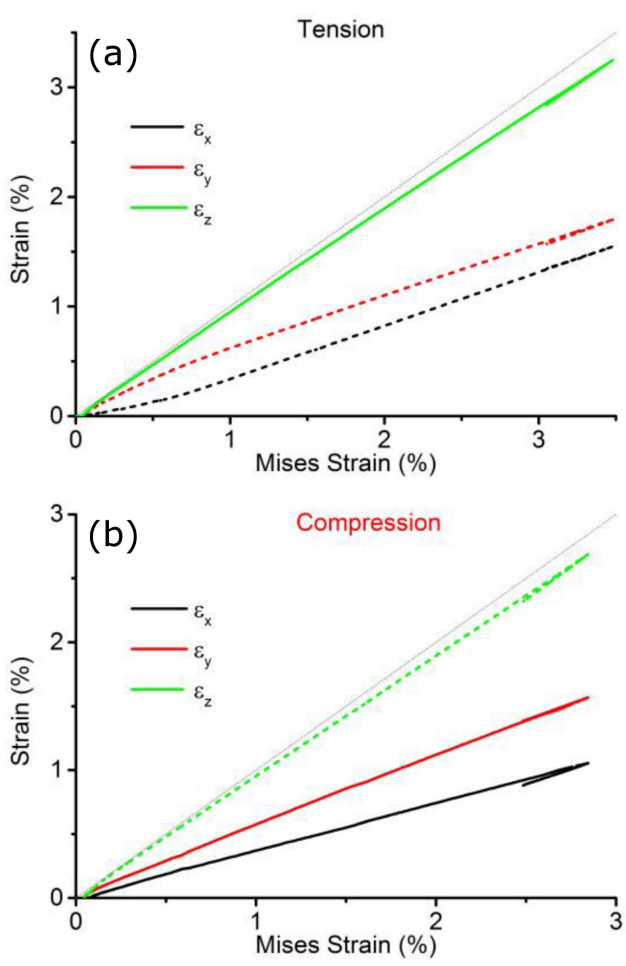

Fig. 4. Comparison of the individual strain components to the Mises strain. Red curve is deformation along axis 1 , black curve along the axis 2 , green along the axis 3 . Solid lines represent the compressive strain, dashed lines tensile strain. Gray line symbolize $1: 1$ ratio.
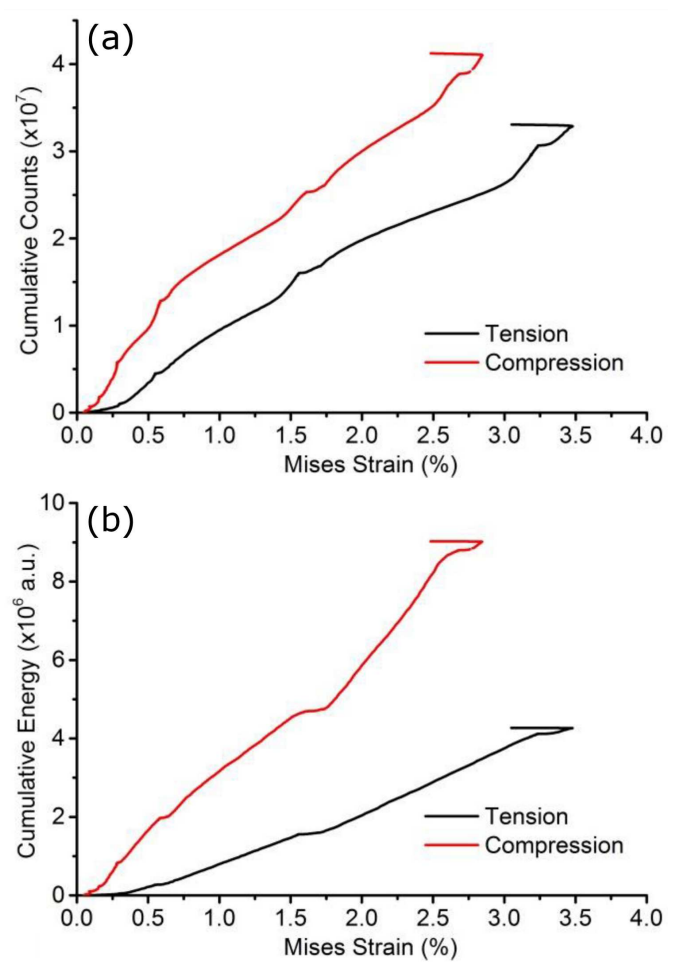

Fig. 5. Cumulative (a) counts, (b) energy of AE events for equibiaxial loading in compression and tension of $\mathrm{Mg}$ specimens as a function of Mises Strain.

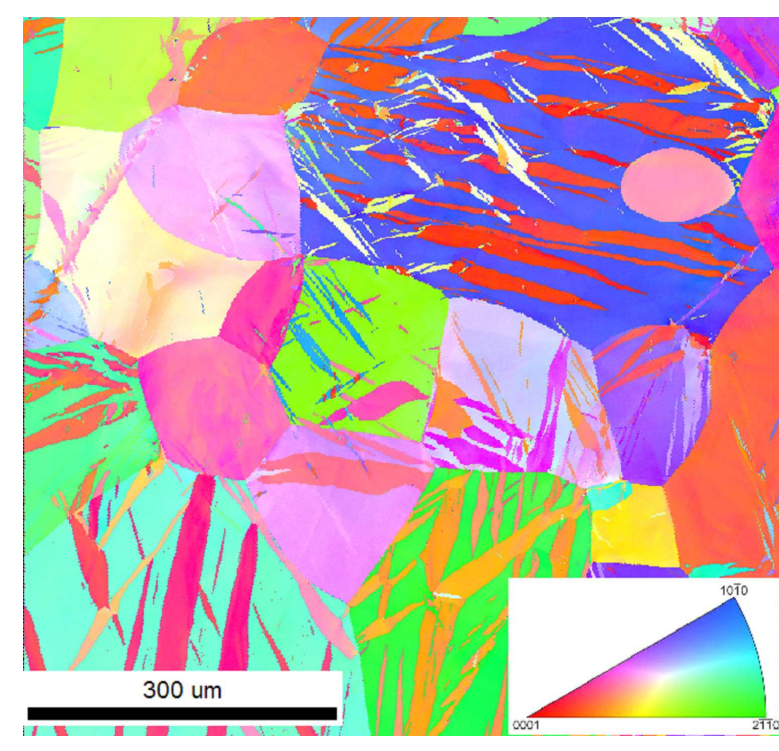

Fig. 6. EBSD map of sample deformed equibiaxially in tension. $Z$-axis is perpendicular to the image plane.

The EBSD map of sample deformed equibiaxially in tension is presented in Fig. 6. In our previous work we have shown that during tension the number of nucleated twin variants is higher than that during the compression. On the other hand the overall twinned volume is larger in compression, owing to the easier growth of twins [7]. Our EBSD investigation revealed that accordingly to our findings, the number of twin variants in grains correspond to the that observed formerly [7] in uniaxial tension. Nevertheless, red colored variants prevail in Fig. 6, which according to our calculations [7] can grow only in the case, if these particular grains undergo compression along the $z$-axis.

\section{Conclusions}

The DIC mapping revealed strong local inhomogeneity of the deformation for $\mathrm{Mg}$ samples with random texture.

The AE suggests that twinning is more pronounced for equibiaxial compression than for equibiaxial tension.

An EBSD map shows that number of nucleated twin variants is a result of the tensile stress; however, the twin growth is driven by the compressive character of the deformation.

\section{Acknowledgments}

The authors are grateful for the financial support of the Czech Science Foundation under the contract 14-36566G. JČ acknowledges the support by the Charles University in Prague, Faculty of Mathematics and Physics, project GA UK Nr. 251715. This work was supported by the project "Nanomaterials centre for advanced applications", Project No. CZ.02.1.01/0.0/0.0/15_003/0000485, financed by ERDF. KAS and TP thank the European Research Council for the financial support within the advanced grant MULTIAX (339245). 


\section{References}

[1] E.W. Kelly, W.F. Hosford, Trans Metall AIME 242 , 654 (1968).

[2] E.O. Hall, Proc Phys Soc London Sect B 64, 747 (1951).

[3] O. Muransky, M.R. Barnett, D.G. Carr, S.C. Vogel, E.C. Oliver, Acta Mater. 58, 1503 (2010).

[4] J. Capek, K. Mathis, B. Clausen, J. Straska, P. Beran, P. Lukas, Mater. Sci. Eng. A 602, 25 (2014).
[5] S. Van Petegem, J. Wagner, T. Panzner, M.V. Upadhyay, T.T.T. Trang, H. Van Swygenhoven, Acta Mater. 105, 404 (2016).

[6] V. Bonnand, J.L. Chaboche, P. Gomez, P. Kanouté, D. Pacou, Int. J. Fatigue 33, 1006 (2011).

[7] J. Čapek, K. Máthis, B. Clausen, M. Barnett, Acta Mater. 130, 319 (2017). 